\title{
LARGE EXCESS OF HEAVY NITROGEN IN BOTH HYDROGEN CYANIDE AND CYANOGEN FROM COMET 17P/HOLMES
}

\author{
D. Bockelée-Morvan, ${ }^{1}$ N. Biver, ${ }^{1}$ E. Jehin, ${ }^{2}$ A. L. Cochran, ${ }^{3}$ H. Wiesemeyer, ${ }^{4}$ J. Manfroid,${ }^{2}$ D. Hutsemékers,${ }^{2}$ \\ C. Arpigny, ${ }^{2}$ J. Boissier,,${ }^{5}$ W. Cochran,${ }^{3}$ P. Colom,${ }^{1}$ J. Crovisier,${ }^{1}$ N. Milutinovic, ${ }^{6}$ R. Moreno, ${ }^{1}$ \\ J. X. Prochaska, ${ }^{7}$ I. Ramirez, ${ }^{3}$ R. Schulz,${ }^{8}$ And J.-M. ZuCCOni ${ }^{9}$ \\ Received 2008 February 6; accepted 2008 April 4; published 2008 April 23
}

\begin{abstract}
From millimeter and optical observations of the Jupiter-family comet 17P/Holmes performed soon after its huge outburst of 2007 October 24 , we derive ${ }^{14} \mathrm{~N} /{ }^{15} \mathrm{~N}=139 \pm 26$ in $\mathrm{HCN}$ and ${ }^{14} \mathrm{~N} /{ }^{15} \mathrm{~N}=165 \pm 40$ in $\mathrm{CN}$, establishing that $\mathrm{HCN}$ has the same nonterrestrial isotopic composition as $\mathrm{CN}$. The same conclusion is obtained for the long-period comet C/1995 O1 (Hale-Bopp) after a reanalysis of previously published measurements. These results are compatible with $\mathrm{HCN}$ being the prime parent of $\mathrm{CN}$ in cometary atmospheres. The ${ }^{15} \mathrm{~N}$ excess relative to the Earth's atmospheric value indicates that N-bearing volatiles in the solar nebula underwent important $\mathrm{N}$ isotopic fractionation at some stage of solar system formation. HCN molecules never isotopically equilibrated with the main nitrogen reservoir in the solar nebula before being incorporated in Oort Cloud and Kuiper Belt comets. The ${ }^{12} \mathrm{C} /{ }^{13} \mathrm{C}$ ratios in $\mathrm{HCN}$ and $\mathrm{CN}$ are measured to be consistent with the terrestrial value.
\end{abstract}

Subject headings: comets: general — comets: individual (17P/Holmes) — radio lines: solar system

\section{INTRODUCTION}

Comets are made of ices, organics, and minerals that are a record of the chemistry of the outer regions of the primitive solar nebula where they agglomerated 4.6 Gyr ago. Compositional analyses of comets can provide important clues to the chemical and physical processes that occurred in the early phases of solar system formation, and in the natal molecular cloud that predated the formation of the solar nebula (Ehrenfreund et al. 2005). In particular, isotopic ratios in cometary volatiles are important diagnostics of how this matter formed, since isotopic fractionation is very sensitive to chemical and physical conditions. However, such measurements are rare. Strong deuterium enhancements are observed in $\mathrm{H}_{2} \mathrm{O}$ and $\mathrm{HCN}$ gases of several comet comae that are characteristic of interstellar or protosolar chemistry at low temperature (Ehrenfreund et al. 2005; Bockelée-Morvan et al. 2005). On the other hand, questions arise as to how to explain the nonterrestrial and uniform values of the ${ }^{14} \mathrm{~N} /{ }^{15} \mathrm{~N}$ isotopic ratio in $\mathrm{CN}$ (Arpigny et al. 2003; Hutsemékers et al. 2005; Manfroid et al. 2005; Jehin et al. 2004), also indicative of interstellar-like chemistry, when this ratio was measured to be terrestrial in HCN (Jewitt et al. 1997; Ziurys et al. 1999), the presumed source of CN radicals in cometary atmospheres. Here we present measurements of the $\mathrm{HC}^{14} \mathrm{~N} / \mathrm{HC}^{15} \mathrm{~N}$ and $\mathrm{H}^{12} \mathrm{CN} / \mathrm{H}^{13} \mathrm{CN}$ isotopic ratios acquired in comet $17 \mathrm{P} /$ Holmes by millimeter spectroscopy, together with

\footnotetext{
${ }^{1}$ LESIA, Observatoire de Paris, 5 Place Jules Janssen, F-92190 Meudon, France; dominique.bockelee@obspm.fr.

${ }^{2}$ Institut d'Astrophysique et de Géophysique, Sart-Tilman, B-4000 Liège, Belgium.

${ }^{3}$ Department of Astronomy and McDonald Observatory, University of Texas at Austin, C-1400, Austin, TX 78712.

${ }^{4}$ IRAM, Avenida Divina Pastora, 7, Núcleo Central, E-18012 Granada, Spain.

${ }^{5}$ IRAM, 300 rue de la Piscine, Domaine Universitaire, F-38406 Saint Martin d'Hères, France.

${ }^{6}$ Department of Physics and Astronomy, University of Victoria, 3800 Finnerty Road, Victoria, BC V8P 5C2, Canada.

${ }^{7}$ Department of Astronomy and Astrophysics, and UCO/Lick Observatory, University of California, 1156 High Street, Santa Cruz, CA 95064.

${ }^{8}$ ESA/RSSD, ESTEC, P.O. Box 299, NL-2200 AG Noordwijk, The Netherlands.

${ }^{9}$ Observatoire de Besançon, F-25010 Besançon Cedex, France.
}

$\mathrm{C}^{14} \mathrm{~N} / \mathrm{C}^{15} \mathrm{~N}$ and ${ }^{12} \mathrm{CN} /{ }^{13} \mathrm{CN}$ measurements from visible spectroscopy. The brightness of this short-period $(6.9 \mathrm{yr})$ comet of the Jupiter family unexpectedly increased (from a total visual magnitude $m_{v}=17$ to $m_{v}=2.5$ ) on 2007 October 24 while it was at a distance of $1.63 \mathrm{AU}$ from the Earth and $2.44 \mathrm{AU}$ from the Sun (Green 2007). This huge outburst of activity, which is likely related to a sudden fragmentation of the nucleus, followed by the subsequent production of a large quantity of grains, offered us the opportunity to search for weak spectral signatures of rare isotopes using complementary techniques.

\section{THE ${ }^{14} \mathrm{~N} /{ }^{15} \mathrm{~N}$ RATIO IN HCN IN 17P/HOLMES}

We carried out observations of 17P/Holmes using the $30 \mathrm{~m}$ telescope of the Institut de Radioastronomie Millimétrique (IRAM) located in the Sierra Nevada (Spain). Isotopic measurements were performed on October 27-28 UT. The tracking of the comet was done using orbital elements K077/06 from the JPL HORIZONS system. The pointing of the telescope was checked and updated by repeated observations of a nearby quasar. Sky cancellation was performed by wobbling the secondary mirror with a throw of $3^{\prime}$ at a rate of $0.5 \mathrm{~Hz}$. Four receivers could be operated at the same time. The $J=3-2$ rotational lines of $\mathrm{H}^{12} \mathrm{C}^{14} \mathrm{~N}$ (hereafter referred to as $\mathrm{HCN}$ ) at 265.9 GHz, $\mathrm{H}^{13} \mathrm{CN}(259.0 \mathrm{GHz})$ and $\mathrm{HC}^{15} \mathrm{~N}(258.2 \mathrm{GHz})$ were measured (Table 1). The beam diameter (half-power beamwidth) of $9.5^{\prime \prime}$ corresponded to $11,300 \mathrm{~km}$ at the distance of the comet. Observations were undertaken in good atmospheric conditions (3-5 $\mathrm{mm}$ precipitable water). The lines were observed both at low (1 or $2 \mathrm{MHz})$ and high $(62 \mathrm{kHz})$ spectral resolutions. Spectra are shown in Figures 1 and 2 , and line areas are given in Table 1. Observations of $\mathrm{HCN}, \mathrm{H}^{13} \mathrm{CN}$, and $\mathrm{HC}^{15} \mathrm{~N}$ were not entirely simultaneous (Table 1). However, several strong lines $\left(\mathrm{CS} J=3-2, \mathrm{CH}_{3} \mathrm{CN} J=8-7\right.$ [147 GHz], and $\mathrm{CH}_{3} \mathrm{OH} J=3-2[145 \mathrm{GHz}$ ] lines) were continuously observed, as a way to monitor the comet's activity. Their intensities decreased by $12 \%$ during the period ( $5.5 \mathrm{hr}$ long) when $\mathrm{HCN}, \mathrm{H}^{13} \mathrm{CN}$, and $\mathrm{HC}^{15} \mathrm{~N}$ data were acquired. This variation was taken into account when deriving the isotopic ratios.

In contrast to the $\mathrm{HC}^{15} \mathrm{~N}$ and $\mathrm{H}^{13} \mathrm{CN}$ lines, $\mathrm{HCN} J=3-2$ is 
TABLE 1

Characteristics of Radio Lines and Molecular Production Rates in Comet 17P/Holmes

\begin{tabular}{|c|c|c|c|c|c|c|c|c|}
\hline Molecule & Line & $\begin{array}{l}\text { Frequency } \\
\quad(\mathrm{GHz})\end{array}$ & $\begin{array}{c}\text { Date UT } \\
\text { (2007 October) }\end{array}$ & $\begin{array}{l}\text { Int. Time } \\
\text { (minutes) }\end{array}$ & $\begin{array}{l}\text { Line Area } \\
\left(\mathrm{K} \mathrm{km} \mathrm{s}^{-1}\right)\end{array}$ & Opacity & $\begin{array}{c}\text { Column Density } \\
\left(10^{12} \text { molecules } \mathrm{cm}^{-2}\right)\end{array}$ & $\begin{array}{c}\text { Production Rate } \\
\left(10^{26} \text { molecules } \mathrm{s}^{-1}\right)\end{array}$ \\
\hline \multirow[t]{3}{*}{$\mathrm{HCN}$} & $J=3-2$ & 265.886434 & $27.96-27.98$ & 15 & $18.23 \pm 0.14$ & 0.56 & $20.8 \pm 0.17$ & $19.85 \pm 0.16$ \\
\hline & $J=3-2, F=2-2$ & 265.888516 & $27.96-27.98$ & 15 & $0.958 \pm 0.083$ & 0.03 & $20.7 \pm 1.8$ & $19.74 \pm 1.71$ \\
\hline & $J=3-2, F=3-3$ & 265.884883 & $27.96-27.98$ & 15 & $1.097 \pm 0.073$ & 0.03 & $23.7 \pm 1.6$ & $22.60 \pm 1.50$ \\
\hline $\mathrm{H}^{13} \mathrm{CN} \ldots \ldots$ & $J=3-2$ & 259.011798 & $28.08-28.19$ & 90 & $0.220 \pm 0.037$ & 0.008 & $1.74 \pm 0.30$ & $0.164 \pm 0.028$ \\
\hline $\mathrm{HC}^{15} \mathrm{~N} \ldots \ldots$ & $J=3-2$ & 258.156996 & $27.96-28.19$ & 145 & $0.191 \pm 0.021$ & 0.006 & $1.51 \pm 0.16$ & $0.140 \pm 0.015$ \\
\hline
\end{tabular}

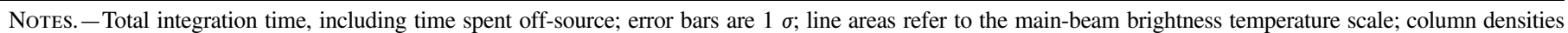

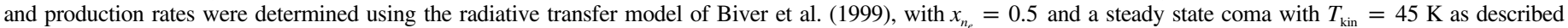

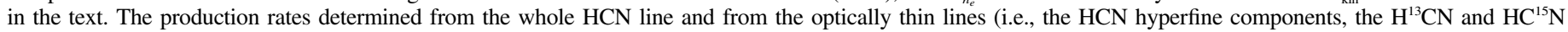
lines) are $6 \%$ lower and 6\% higher, respectively, using $T_{\text {kin }}=65 \mathrm{~K}$ derived by Dello Russo et al. (2008) from HCN infrared observations on 2007 October 27.6 .

optically thick (Table 1). Therefore, radiative transfer modeling is required to retrieve the $\mathrm{HCN} / \mathrm{H}^{13} \mathrm{CN}$ and $\mathrm{HCN} / \mathrm{HC}^{15} \mathrm{~N}$ isotopic ratios if the $\mathrm{HCN} J=3-2$ line is used in the analysis. The coma of comet Holmes was in a nonequilibrium regime following its outburst, with gas-phase species released by icy grains. Unavoidable simplifying model assumptions on the coma structure could introduce systematic opacity-dependent errors in the retrievals and affect the determination of the isotopic ratios. However, the HCN $J=3-2$ line is split into six hyperfine components, two of which $(F=2-2$ and $F=3-3)$ are well separated from the core of the line and are detected in the $\mathrm{HCN}$ spectrum (Fig. 2; Table 1). These hyperfine components have intrinsic line strengths of $3.7 \%$ the total strength, according to hyperfine statistical weights, and are optically thin (Table 1 ). The $\mathrm{HCN} / \mathrm{H}^{13} \mathrm{CN}$ and $\mathrm{HCN} / \mathrm{HC}^{15} \mathrm{~N}$ abundance ratios, determined using the $\mathrm{HCN}$ optically thin hyperfine components, do not depend on model assumptions on coma temperature, structure, and temporal variability, because, in this case, emission lines from molecules in the same excitation state and in the same regions of the coma are compared. They are directly given by the line intensity ratios, albeit with minor corrections that account for the slightly different line frequencies. Using the $\mathrm{HCN}$ hyperfine components, and correcting for the nonsimultaneity of the $\mathrm{HCN}, \mathrm{H}^{13} \mathrm{CN}$, and $\mathrm{HC}^{15} \mathrm{~N}$ measurements, we derive $\mathrm{H}^{12} \mathrm{CN} / \mathrm{H}^{13} \mathrm{CN}=114 \pm 26$ and $\mathrm{HC}^{14} \mathrm{~N} / \mathrm{HC}^{15} \mathrm{~N}=139 \pm 26$.

Column densities and production rates determined with the radiative transfer model of Biver et al. (1999) are given in Table 1. We assumed a steady state isotropic parent molecule distribution, with a gas velocity of $0.56 \mathrm{~km} \mathrm{~s}^{-1}$ and a gas kinetic temperature $T_{\text {kin }}$ of $45 \mathrm{~K}$ inferred from IRAM observations of multiple lines of $\mathrm{CH}_{3} \mathrm{OH}$ on October 29.0 UT. Interestingly, isotopic ratios obtained using the $\mathrm{HCN}$ production rate deduced from the whole $J=3-2$ line are similar to those obtained using the model-independent hyperfine line method. As shown

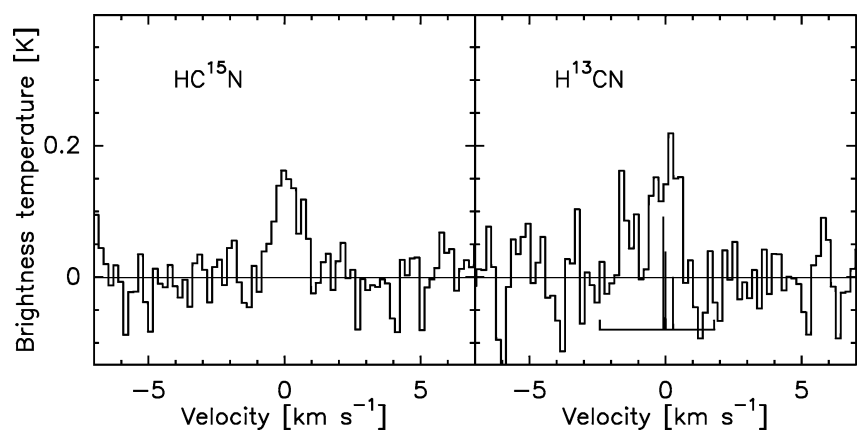

FIG. 1.-Spectra of the $J=3-2$ lines of $\mathrm{HC}^{15} \mathrm{~N}$ and $\mathrm{H}^{13} \mathrm{CN}$ in comet $17 \mathrm{P} /$ Holmes on 2007 October $27-28$. The velocity frame is with respect to the comet rest velocity. The positions and relative intensities of the hyperfine components of $\mathrm{H}^{13} \mathrm{CN} J=3-2$ are shown. in Figure 2, when the dayside and nightside $\mathrm{HCN}$ velocities are fixed to 0.6 and $0.4 \mathrm{~km} \mathrm{~s}^{-1}$, the model provides a satisfactory fit to the shape of the HCN line. This suggests that our description of the HCN spatial distribution and excitation is correct in first approximation. This conclusion is supported by the good agreement between the $\mathrm{HCN}$ production rate measured on October 27.6 in a smaller $\left(\sim 1^{\prime \prime}\right)$ aperture (Dello Russo et al. 2008) and those reported in this work.

\section{THE ${ }^{14} \mathrm{~N} /{ }^{15} \mathrm{~N}$ RATIO IN CN IN 17P/HOLMES}

High-resolution optical observations were performed to measure the ${ }^{14} \mathrm{~N} /{ }^{15} \mathrm{~N}$ and ${ }^{12} \mathrm{C} /{ }^{13} \mathrm{C}$ ratios in $\mathrm{CN}$. Spectra of the $B$ ${ }^{2} \Sigma^{+}-X^{2} \Sigma^{+}(0,0) \mathrm{CN}$ band at $388 \mathrm{~nm}$ were obtained on October $25.4,28.3,29.4,30.4$, and 31.4 and on November 18.4 and 19.32007 UT with the 2D coude spectrograph at the $2.7 \mathrm{~m}$ Harlan J. Smith telescope of the McDonald Observatory. A series of short exposures, from $30 \mathrm{~s}$ to 5 minutes, were also collected on 2007 October 29.6 UT with the High Resolution Echelle Spectrometer of the Keck I telescope installed on Mauna Kea (Hawaii). The observations were carried out under clear weather and low air mass. In both cases, the slit of the spectrograph was $\sim 1^{\prime \prime}$ wide and $\sim 7^{\prime \prime}$ long, providing a resolving power of about $\lambda / \Delta \lambda=60,000\left(0.03 \AA\right.$ pixel $\left.^{-1}\right)$. The slit was centered on the false nucleus in the case of the Keck spectra and displaced in the coma (by up to $20^{\prime \prime}$ ) for the McDonald exposures ( 20 minutes), to reduce the contamination by the strong dust-reflected spectrum. The dust-reflected sunlight underlying the spectral lines [among which are rovibrational lines of the ${ }^{12} \mathrm{C}^{14} \mathrm{~N},{ }^{13} \mathrm{C}^{14} \mathrm{~N}$, and ${ }^{12} \mathrm{C}^{15} \mathrm{~N}(0,0)$ band] was removed by subtracting a solar reference spectrum after the appropriate Doppler shift, profile fitting, and normalization were applied (Arpigny et al. 2003; Jehin et al. 2004). The individual CN (0, 0 ) spectra were then combined with an optimal weighting

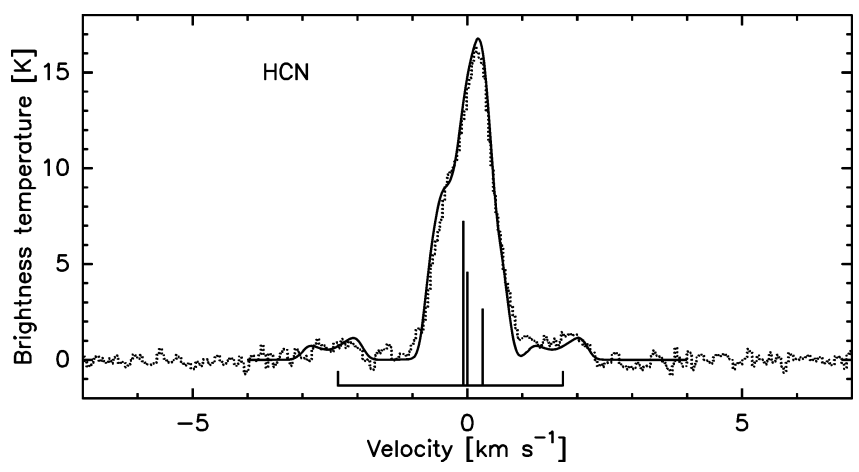

FIG. 2.-Model fit (solid line; see text) to the $J=3-2 \mathrm{HCN}$ line profile observed in comet 17P/Holmes on October 27.97 UT (dotted line). Positions and relative intensities of the hyperfine components are shown. 
scheme in order to maximize the overall signal-to-noise ratio. Synthetic spectra of ${ }^{12} \mathrm{C}^{14} \mathrm{~N},{ }^{13} \mathrm{C}^{14} \mathrm{~N}$, and ${ }^{12} \mathrm{C}^{15} \mathrm{~N}$ were computed for each observing circumstance using a fluorescence model (Zucconi \& Festou 1985). We took into account slightly different excitation conditions over the period of the observations caused by small variations of the heliocentric distance and velocity. Collisional effects were empirically estimated by fitting the ${ }^{12} \mathrm{C}^{14} \mathrm{~N}$ lines (Manfroid et al. 2005). The synthetic spectra were then co-added in the same way as the data. The isotope mixture was adjusted to best fit the observed continuum-subtracted spectrum. We considered seven $R$-branch lines $(R 3-R 9)$, as shown in Figure 3. The isotopic ratios of ${ }^{12} \mathrm{C} /{ }^{13} \mathrm{C}$ and ${ }^{14} \mathrm{~N} /{ }^{15} \mathrm{~N}$ in the final co-added Keck and McDonald spectrum are estimated to be $90 \pm 20$ and $165 \pm 40$, respectively.

\section{REANALYSIS OF THE COMET HALE-BOPP DATA}

The ${ }^{14} \mathrm{~N} /{ }^{15} \mathrm{~N}$ ratios measured in $\mathrm{HCN}$ and $\mathrm{CN}$ in comet $17 \mathrm{P} /$ Holmes are consistent with each other. In contrast, an $\mathrm{HC}^{14} \mathrm{~N} /$ $\mathrm{HC}^{15} \mathrm{~N}$ ratio marginally higher than the Earth's atmospheric value (272) was reported for comet C/1995 O1 (Hale-Bopp) (Jewitt et al. 1997; Ziurys et al. 1999), whereas the ${ }^{14} \mathrm{~N} /{ }^{15} \mathrm{~N}$ value measured in $\mathrm{CN}$ is $140 \pm 35$ (Arpigny et al. 2003). This led us to reanalyze the measurements made in comet Hale-Bopp.

Our reanalysis of the data of Ziurys et al. (1999) obtained on 1997 March 24 and 25 yields ${ }^{12} \mathrm{C} /{ }^{13} \mathrm{C}=65 \pm 13$ and ${ }^{14} \mathrm{~N} /$ ${ }^{15} \mathrm{~N}=152 \pm 30$ (with a $10 \%$ calibration uncertainty included), as compared with the $\mathrm{HC}^{14} \mathrm{~N} / \mathrm{HC}^{15} \mathrm{~N}$ production rate ratios of $100 \pm 20$ and $286 \pm 82$ given by these authors. Ziurys et al. used approximate formulas to analyze the optically thick HCN lines, assuming, in addition, an inappropriate value for the rotational temperature of $\mathrm{HCN}$. Instead, we used full radiative transfer modeling. Our determinations are likely more reliable, as the $\mathrm{HCN}$ production rates that we derive from the $J=1-$ 0, 2-1, and 3-2 HCN lines observed on March 24 are consistent within $5 \%$, whereas the values inferred by Ziurys et al. from the different lines differ by up to a factor of 2 . The $\mathrm{HC}^{15} \mathrm{~N}$ and $\mathrm{H}^{13} \mathrm{CN} J=3-2$ lines were observed on March 25. As one cannot exclude day-to-day variations in $\mathrm{HCN}$ production from March 24 to 25 , the $\mathrm{HC}^{15} \mathrm{~N} / \mathrm{H}^{13} \mathrm{CN}$ ratio of $0.43 \pm 0.10$, deduced from March 25 data only, could be more secure. In this case, assuming ${ }^{12} \mathrm{C} /{ }^{13} \mathrm{C}$ to be equal to the terrestrial value of 89 , we deduce ${ }^{14} \mathrm{~N} /{ }^{15} \mathrm{~N}=207 \pm 48$.

The data obtained by Jewitt et al. (1997) at the James Clerk Maxwell Telescope (JCMT) are public and available from the Canadian Astronomy Data Centre. Reanalyzing these data, we found the following: (1) The HCN data were acquired during nighttime near sunrise, whereas $\mathrm{H}^{13} \mathrm{CN}$ and especially $\mathrm{HC}^{15} \mathrm{~N}$ were observed later during daytime; the beam efficiency may have degraded by $20 \%$ according to JCMT specifications. (2) The HCN line was observed with the receiver B3 tuned in double sideband (DSB), whereas the other lines were observed in single sideband; HCN spectra of calibration sources obtained with the same receiver DSB tuning as used for the cometary observations show signals in excess of $15 \%$ with respect to reference spectra of the sources. (3) The $\mathrm{H}^{13} \mathrm{CN} J=4-3$ line is blended with the $\mathrm{SO}_{2}$ $13_{2,12}-12_{1,11}$ line at $345.338538 \mathrm{GHz}$ (Lis et al. 1997); using the $\mathrm{SO}_{2} / \mathrm{HCN}$ production rate ratio determined for comet Hale-Bopp (Bockelée-Morvan et al. 2000), we estimate that it affects the $\mathrm{H}^{13} \mathrm{CN}$ line intensity by $20 \%$. (4) More critically, the $\mathrm{HC}^{15} \mathrm{~N}$ spectra present scan-to-scan intensity variations by a factor of 2 (a factor of 10 above the fluctuations related to the statistical noise) that are likely of instrumental origin. Taking these corrections into account, we infer ${ }^{12} \mathrm{C} /{ }^{13} \mathrm{C}=94 \pm 8$ and ${ }^{14} \mathrm{~N} /{ }^{15} \mathrm{~N}=205 \pm 70$,

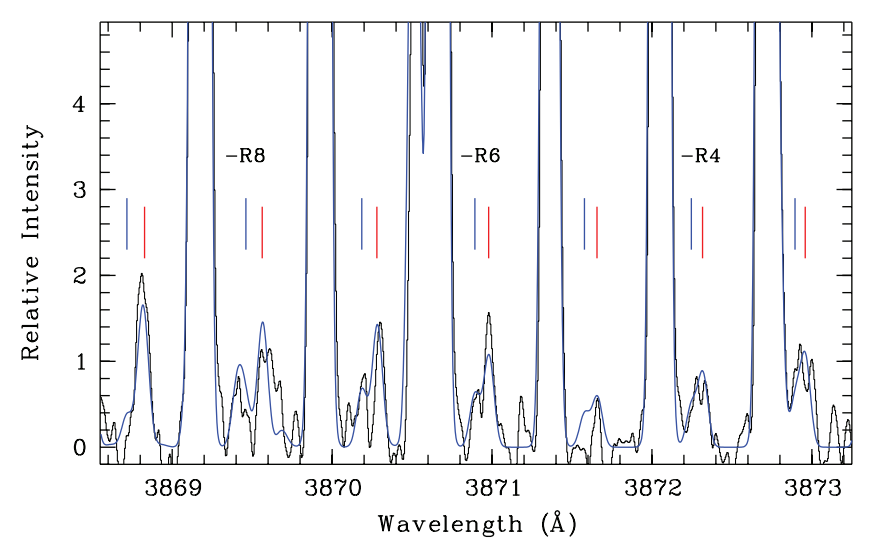

FIG. 3.-Small part of the co-added Keck and McDonald CN $(0,0)$ band spectra of comet $17 \mathrm{P} /$ Holmes showing seven $R$-branch lines $(R 3-R 9)$ of $\mathrm{CN}$ isotopes (black line). The dust-scattered solar spectrum was removed. The positions of the ${ }^{13} \mathrm{C}^{14} \mathrm{~N}$ (red ticks) and ${ }^{12} \mathrm{C}^{15} \mathrm{~N}$ (blue ticks) lines are indicated; the corresponding lines of the main isotope are on the left. The synthetic spectrum with ${ }^{12} \mathrm{C} /{ }^{13} \mathrm{C}=90$ and ${ }^{14} \mathrm{~N} /{ }^{15} \mathrm{~N}=165$ is superimposed (blue line).

whereas the values given in Jewitt et al. (1997) are $100 \pm 12$ and $323 \pm 46$, respectively. The large uncertainty in our ${ }^{14} \mathrm{~N} /{ }^{15} \mathrm{~N}$ determination reflects the dispersion of the $\mathrm{HC}^{15} \mathrm{~N}$ measurements.

We conclude that the ${ }^{14} \mathrm{~N} /{ }^{15} \mathrm{~N}$ ratio in $\mathrm{HCN}$ is rather uncertain for comet Hale-Bopp but is consistent with the value measured in $\mathrm{CN}$.

\section{IMPLICATIONS}

The ${ }^{12} \mathrm{C} /{ }^{13} \mathrm{C}$ values in $\mathrm{HCN}$ and $\mathrm{CN}$ are in agreement with the terrestrial value of 89 and previous measurements in comets (e.g., Bockelée-Morvan et al. 2005).

The ${ }^{14} \mathrm{~N} /{ }^{15} \mathrm{~N}$ ratios measured in $\mathrm{HCN}$ and $\mathrm{CN}$ in comet $17 \mathrm{P} /$ Holmes both correspond to a factor of $2{ }^{15} \mathrm{~N}$ enrichment relative to the Earth's atmospheric value and are consistent with the ${ }^{14} \mathrm{~N} /{ }^{15} \mathrm{~N}$ ratios measured in $\mathrm{CN}$ in a dozen comets that cluster at $141 \pm 29$ (Arpigny et al. 2003; Hutsemékers et al. 2005; Manfroid et al. 2005; Jehin et al. 2004). The discrepancy between HCN and $\mathrm{CN}$ isotopic ratios previously found for comet Hale-Bopp led to the suggestion of a $\mathrm{CN}$ production mechanism other than $\mathrm{HCN}$ photolysis in cometary atmospheres, possibly from the thermal degradation of ${ }^{15} \mathrm{~N}$-rich refractory organics present in dust grains. This interpretation was supported by the presence of $\mathrm{CN}$ jets, by the radial distribution of $\mathrm{CN}$, found to be generally less extended than expected from $\mathrm{HCN}$ photodissociation, and by the $\mathrm{CN} / \mathrm{HCN}$ production rate ratio, which exceeds unity in some comets (Fray et al. 2005). However, questions arose as to how to explain the equally low $\mathrm{C}^{14} \mathrm{~N} / \mathrm{C}^{15} \mathrm{~N}$ value observed in comet Hale-Bopp at large heliocentric distance, where the $\mathrm{CN}$ radicals were expected to be mainly HCN photodissociation products (Manfroid et al. 2005; Rauer et al. 2003). Our reanalysis of the Hale-Bopp data, which shows that the ${ }^{14} \mathrm{~N} /{ }^{15} \mathrm{~N}$ ratio in $\mathrm{HCN}$ encompasses the $\mathrm{CN}$ value, solves this issue. Also, the similar isotopic ratios found in $\mathrm{HCN}$ and $\mathrm{CN}$ provide a better explanation for the uniform values of the $\mathrm{C}^{14} \mathrm{~N} / \mathrm{C}^{15} \mathrm{~N}$ ratio among comets exhibiting large differences in dust-to-gas production rate ratios.

Our isotopic measurements are compatible with HCN being the prime parent of $\mathrm{CN}$ in cometary atmospheres. For comet HaleBopp, the production rates of the two species were found to be approximately equal (Rauer et al. 2003; Fray et al. 2005). The complex and variable structure of 17P/Holmes's coma after its outburst makes comparisons of the $\mathrm{HCN}$ and $\mathrm{CN}$ production rates 
difficult for this comet. However, Dello Russo et al. (2008) note that the good agreement between the $\mathrm{HCN} / \mathrm{H}_{2} \mathrm{O}$ abundance determined from infrared spectra and the $\mathrm{OH} / \mathrm{CN}$ abundance ratio measured with narrowband photometry (Schleicher 2007) is consistent with $\mathrm{CN}$ being mainly produced by $\mathrm{HCN}$ photolysis. Yet we cannot exclude that, in other comets, $\mathrm{CN}$ has other major progenitors (dust or gas-phase species) sharing the same low ${ }^{14} \mathrm{~N} /$ ${ }^{15} \mathrm{~N}$ isotopic ratio, which would mean that ${ }^{15} \mathrm{~N}$ enrichment is possibly a general property of $\mathrm{CN}$-bearing compounds in comets.

Comets Hale-Bopp and 17P/Holmes are of different dynamical families, the former originating from the Oort Cloud and the latter from the trans-Neptunian scattered disk. An anomalous nitrogen isotopic composition in $\mathrm{CN}$ is observed in a number of comets from these two dynamical populations. Hence, comets issued from these two reservoirs likely exhibit similar anomalous $\mathrm{N}$ isotopic composition in $\mathrm{HCN}$.

The ${ }^{15} \mathrm{~N}$ excess measured in cometary $\mathrm{HCN}\left(\delta^{15} \mathrm{~N} \sim 1000 \%\right.$ o relative to the Earth's atmospheric value) and the $\mathrm{CN}$ daughter product cannot result from isotopic fractionation in the comet atmosphere. It is comparable to the extreme enrichments measured in interplanetary dust particles (IDPs; Floss et al. 2006) and carbonaceous meteorites (Busemann et al. 2006). High $\delta^{15} \mathrm{~N}$ values are also present at the submicrometer scale in the dust particles collected by the Stardust mission from comet 81P/Wild 2 (McKeegan et al. 2006). In IDPs and meteorites, the ${ }^{15} \mathrm{~N}$-rich nitrogen is carried by nonvolatile macromolecular organic material and is generally believed to be a remnant of interstellar chemistry, although a nucleosynthesis source is often considered as an alternative mechanism given the presence of presolar grains with high ${ }^{15} \mathrm{~N}$ excesses in the matrices of meteorites (e.g., Zinner 1998). These complex organics possibly formed from UV or cosmic radiation processing of simple ices in the presolar cloud or, at later stages, in the cold regions of the solar nebula. The enrichments reported here provide the first evidence for the presence of high ${ }^{15} \mathrm{~N}$ anomalies in the volatiles that composed the icy phase of the outer solar nebula, possibly representing the precursors of the primitive refractory organics.

Cometary $\mathrm{HCN}$ and $\mathrm{H}_{2} \mathrm{O}$ ices exhibit strong enrichments in deuterium with respect to the cosmic D/H value (Ehrenfreund et al. 2005; Bockelée-Morvan et al. 2005), and these enrichments are believed to reflect ion-molecule and gas-grain fractionation reactions that took place at low temperature in the early phases of solar system formation or in the natal molecular cloud. The interpretation of the ${ }^{15} \mathrm{~N}$ enrichment in $\mathrm{HCN}$ (by a factor of 3 with respect to the protosolar value in the main nitrogen reservoir; Fouchet et al. 2004; Meibom et al. 2007) is not as compelling as it is for deuterium, because there is still little evidence for $\mathrm{N}$ isotopic fractionation in the interstellar medium (Ikeda et al. 2002) and because predicted ${ }^{15} \mathrm{~N}$ enhancements in $\mathrm{HCN}$ for exchange reactions involving the main nitrogen reservoir $\mathrm{N}_{2}$ are modest (Terzieva \& Herbst 2000). Rodgers \& Charnley $(2004,2008)$ show that highly fractionated $\mathrm{NH}_{3}$ ice could have formed in interstellar or protosolar material if $\mathrm{N}_{2}$ was converted into atomic nitrogen. Whereas this mechanism is attractive for accounting for the high ${ }^{15} \mathrm{~N}$ excesses in primitive refractory organics, if synthesized from $\mathrm{NH}_{3}$, the chemical network for explaining the $\mathrm{HCN}$ isotopic anomaly in comets still has to be proposed. Alternative mechanisms include a nucleosynthetic origin and photochemical self-shielding in the solar nebula, similar to the mechanism proposed for explaining the oxygen isotope anomalies in meteorites (Clayton 2002a, 2002b). Irrespective of the mechanism, protosolar HCN never isotopically equilibrated with nebular $\mathrm{N}_{2}$ at the later phases of the evolution of the solar system.

IRAM is supported by INSU/CNRS (France), MPG (Germany), and IGN (Spain). We thank the IRAM staff, and especially P. Cox, for providing us with discretionary director time, and $\mathrm{C}$. Thum, for having scheduled these observations on short notice. Some of the data presented herein were obtained at the W. M. Keck Observatory, which is operated as a scientific partnership among the California Institute of Technology, the University of California, and the National Aeronautics and Space Administration. The Observatory was made possible by the generous financial support of the W. M. Keck Foundation. This Letter includes data taken at the McDonald Observatory of the University of Texas at Austin. We thank G. Punawai, for his invaluable assistance during the observing run at Keck I, and E. Lellouch and D.C. Lis, for helpful comments on the manuscript. J. M. is Research Director, E. J. is Research Associate, and D.H. is Senior Research Associate at FNRS (Belgium). A. L. C. was supported by NASA grant NNG04G162G.

Facilities: IRAM:30m, Keck:I, McD:2.7m.

\section{REFERENCES}

Arpigny, C., Jehin, E., Manfroid, J., Hutsemékers, D., Schulz, R., Stüwe, J. A., Zucconi, J.-M., \& Ilyin, I. 2003, Science, 301, 1522

Biver, N., et al. 1999, AJ, 118, 1850

Bockelée-Morvan, D., Crovisier, J., Mumma, M. J., \& Weaver, H. A. 2005, in Comets II, ed. M. C. Festou, H. U. Keller, \& H. A. Weaver (Tucson: Univ. Arizona Press), 391

Bockelée-Morvan, D., et al. 2000, A\&A, 353, 1101

Busemann, H., Young, A. F., Alexander, C. M. O'D., Hoppe, P., Mukhopadhyay, S., \& Nittler, L. R. 2006, Science, 312, 727

Clayton, R. N. 2002a, Meteoritics Planet. Sci., 37, A35 2002b, Nature, 415, 860

Dello Russo, N., Vervack, R. J., Jr., Weaver, H. A., Montgomery, M. M., Deshpande, R., Fernandez, Y. R., \& Martin, E. L. 2008, ApJ, in press

Ehrenfreund, P., Charnley, S. B., \& Wooden, D. 2005, in Comets II, ed. M. C. Festou, H. U. Keller, \& H. A. Weaver (Tucson: Univ. Arizona Press), 115

Floss, C., Stadermann, F. J., Bradley, J. P., Dai, Z. R., Bajt, S., Graham, G., \& Lea, A. S. 2006, Geochim. Cosmochim. Acta, 70, 2371

Fouchet, T., Irwin, P. G. J., Parrish, P., Calcutt, S. B., Taylor, F. W., Nixon, C. A., \& Owen, T. 2004, Icarus, 172, 50

Fray, N., Bénilan, Y., Cottin, H., Gazeau, M.-C., \& Crovisier, J. 2005, Planet. Space Sci., 53, 1243
Green, D. W. E. 2007, IAU Circ. 8886

Hutsemékers, D., Manfroid, J., Jehin, E., Arpigny, C., Cochran, A., Schulz, R., Stüwe, J. A., \& Zucconi, J.-M. 2005, A\&A, 440, L21

Ikeda, M., Hirota, T., \& Yamamoto, S. 2002, ApJ, 575, 250

Jehin, E., et al. 2004, ApJ, 613, L161

Jewitt, D., Matthews, H. E., Owen, T., \& Meier, R. 1997, Science, 278, 90

Lis, D. C., et al. 1997, Icarus, 130, 355

Manfroid, J., Jehin, E., Hutsemékers, D., Cochran, A., Zucconi, J.-M., Arpigny, C., Schulz, R., \& Stüwe, J. A. 2005, A\&A, 432, L5

McKeegan, K. D., et al. 2006, Science, 314, 1724

Meibom, A., Krot, A.N., Robert, F., Mostefaoui, S., Russel, S., Petaec, M., \& Gounelle, M. 2007, ApJ, 656, L33

Rauer, H., et al. 2003, A\&A, 397, 1109

Rodgers, S. D., \& Charnley, S. B. 2004, MNRAS, 352, 600 2008, MNRAS, 385, L48

Schleicher, D. G. 2007, IAU Circ. 8889

Terzieva, R., \& Herbst, E. 2000, MNRAS, 317, 563

Zinner, E. 1998, Annu. Rev. Earth Planet. Sci., 26, 147

Ziurys, L. M., Savage, C., Brewster, M. A., Apponi, A. J., Pesch, T. C., \& Wyckoff, S. 1999, ApJ, 527, L67

Zucconi, J. M., \& Festou, M. C. 1985, A\&A, 150, 180 